\title{
Success Rate of Zirconia Crowns over Stainless Steel Crowns in Primary Molars: A Systematic Review and Meta-analysis
}

\author{
Padmapriya $\mathrm{S}^{1}$, Srinath Krishnappa ${ }^{2}$, Sahana Srinath ${ }^{3}$
}

\begin{abstract}
Research question: Are zirconia crowns clinically superior to stainless steel crown in restoring primary molars.

Materials and methods: Articles in electronic databases from 2000 to 2020 via PubMed, Google Scholar, Science Direct and Cochrane Central were searched by 2 reviewers and full text of the article was included for assessment based on inclusion and exclusion criteria. Electronic data base search was complemented by manual search of reference of the entire selected full-text article. Study design, sample size, criteria for evaluation and follow-up period were assessed.

Results: Search yielded 4,609 titles and 61 articles were considered for full text. Seven randomized control trials on primary molars were included in the review, with follow-up from 12 months to 36 months. Studies have evaluated restorative success and gingival health in zirconia and stainless steel groups. Only one study evaluated radiographic success rate of zirconia and stainless steel crowns in primary molars. As the follow-up period progressed, no plaque accumulation was seen on both the zirconia and stainless steel groups. Six articles were considered for meta-analysis. The study data was found to be heterogeneous and random effects model was used. No differences were found in clinical success rate between zirconia and stainless steel group.

Conclusion: Gingival health was superior for zirconia crowns when compared to stainless steel crowns. However clinically, stainless steel crowns are as effective as zirconia crowns.
\end{abstract}

Keywords: Primary molars, Stainless steel crowns, Zirconia crowns.

Journal of South Asian Association of Pediatric Dentistry (2021): 10.5005/jp-journals-10077-3094

\section{INTRODUCTION}

Early childhood caries is a multifactorial disease which involves maxillary primary incisors and rapidly spread to other teeth leading to cavitation. If untreated the caries rapidly progress to dental pulp resulting in pulpitis and even life threatening facial infections.

Stainless steel crowns are widely used for primary molars after endodontic treatment. Other indications are multi-surface caries, hypoplastic teeth, fractured teeth, bruxism and open bite. ${ }^{2}$ Stainless steel crowns are treatment of choice due to its full coverage and less microleakage. ${ }^{3}$

However, a higher esthetic concerns are raised by the parents which increased the demand for tooth colored restorations and it has become a parameter for choosing a primary dental restoration. ${ }^{4}$

Ceramics developed for dental and medical purposes are broadly classified as bioceramic materials. Introduction of zirconia have paved way for an era of metal free dentistry. The name "Zirconium" has been taken from the Arabic word "Zargon" which means "Golden in color". 5

Zirconium oxide crystals exist in three crystallographic phases: the cubic phase $(C)$, the tetragonal phase $(T)$ and the monoclinic phase (M). The cubic phase is stable above $2370^{\circ} \mathrm{C}$. The tetragonal phase is stable between $1170^{\circ} \mathrm{C}$ and $2370{ }^{\circ} \mathrm{C}$ and has improved mechanical properties. The monoclinic phase is stable at room temperature up to $1170{ }^{\circ} \mathrm{C}$ and has lower mechanical properties. $^{6}$

Zirconia crowns were introduced to Pediatric dentistry, recently. Zirconia is a crystalline dioxide of zirconium with mechanical properties, similar to that of a metal and color matches to that of a

\begin{abstract}
1-2Pediatric and Preventive Dentistry, Government Dental College and Research Institute, Bengaluru, Karnataka, India

${ }^{3}$ Oral Pathology, Government Dental College and Research Institute Bengaluru, Karnataka, India

Corresponding Author: Padmapriya S, Pediatric Dentistry, Government Dental College and Research Institute, Bengaluru, Karnataka, India, Phone: + 91 9497293318, e-mail: padmapriya.vtm@gmail.com

How to cite this article: Padmapriya S, Krishnappa S, Srinath S. Success Rate of Zirconia Crowns over Stainless Steel Crowns in Primary Molars: A Systematic Review and Meta-analysis. J South Asian Assoc Pediatr Dent 2021;4(3):212-218.
\end{abstract}

Source of support: $\mathrm{Nil}$

Conflict of interest: None

tooth. Preformed zirconia crowns are available for primary incisors and molars. ${ }^{7}$

Zirconia crown is the topic of current interest in pediatric dentistry and number of articles were published in the literature comparing the zirconia crowns with the stainless steel crowns. This review mainly concentrates on success rates of zirconia crowns in terms of gingival health and restorative success. The goal of this review is to summarize data on clinical performance and to aid in decision making process on choosing stainless steel crowns and zirconia crowns appropriately.

\section{Materials and Methods}

Systematic review and meta-analysis was registered in International Prospective Register of Systematic Reviews, Prospero (CRD42021231526).

() The Author(s). 2021 Open Access This article is distributed under the terms of the Creative Commons Attribution 4.0 International License (https://creativecommons. org/licenses/by-nc/4.0/), which permits unrestricted use, distribution, and non-commercial reproduction in any medium, provided you give appropriate credit to the original author(s) and the source, provide a link to the Creative Commons license, and indicate if changes were made. The Creative Commons Public Domain Dedication waiver (http://creativecommons.org/publicdomain/zero/1.0/) applies to the data made available in this article, unless otherwise stated. 


\section{Search Strategy}

Search strategy was done by two reviewers (Reviewer PS and SK) independently for articles from 2000 to 2020 via PubMed, Google Scholar, Science Direct and Cochrane Central. Articles published in English were included with following keywords: "Zirconia crowns," "Primary tooth," "Crowns in primary molars," "Zirconium oxide," "Ceramics" and "All ceramics." Titles of the articles were screened initially followed by a screening of abstracts. Full-text articles reporting clinical trials were included in the review and were assessed based on inclusion and exclusion criteria. Any disagreement in the screening and selection process among the authors was resolved by discussion. If not solved, arbitration was done with the help of an independent reviewer (Reviewer NS). Reason for excluding the study was recorded. The data obtained was tabulated and narrated. Article screening and selection were summarized in Figure 1. Study design, sample size, criteria for evaluation and follow up period were assessed. The electronic search was complimented by manual search. Bibliographies of retrieved full text articles and reviews that reported on the zirconia crowns were searched manually. Search details in PubMed are as follows:

("zirconia s"[All Fields] OR "zirconias"[All Fields] OR "zirconium oxide"[Supplementary Concept] OR "zirconium oxide"[All Fields] OR "zirconia"[All Fields]) AND ("crown s"[All Fields] OR "crowned"[All Fields] OR "crowns"[MeSH Terms] OR "crowns"[All Fields] OR "crown"[All Fields]) AND (("primaries"[All Fields] OR "primary"[All Fields]) AND ("molar"[MeSH Terms] OR "molar"[All Fields] OR "molars"[All Fields] OR "molar s"[All Fields]))

("ceram"[All Fields] OR "ceramics"[MeSH Terms] OR "ceramics"[All Fields] OR "ceramic"[All Fields] OR "ceramization"[All Fields] OR "cerammed"[All Fields] OR "ceramming"[All Fields]) AND (("primaries"[All Fields] OR "primary"[All Fields]) AND ("molar"[MeSH Terms] OR "molar"[All Fields] OR "molars"[All Fields] OR "molar s"[All Fields])) AND ("crown s"[All Fields] OR "crowned"[All Fields] OR "crowns"[MeSH Terms] OR "crowns"[All Fields] OR "crown"[All Fields])

"zirconia s"[All Fields] OR "zirconias"[All Fields] OR "zirconium oxide"[Supplementary Concept] OR "zirconium oxide"[All Fields] OR "zirconia"[All Fields]) AND ("stainless steel"[MeSH Terms] OR ("stainless"[All Fields] AND "steel"[All Fields]) OR "stainless steel"[All Fields]) AND ("crown s"[All Fields] OR "crowned"[All Fields] OR "crowns"[MeSH Terms] OR "crowns"[All Fields] OR "crown"[All Fields]) AND (("deciduous"[All Fields] OR "deciduousness"[All Fields]) AND ("molar"[MeSH Terms] OR "molar"[All Fields] OR "molars"[All Fields] OR "molar s"[All Fields]))

("zirconia s"[All Fields] OR "zirconias"[All Fields] OR "zirconium oxide"[Supplementary Concept] OR "zirconium oxide"[All Fields] OR "zirconia"[All Fields]) AND ("crown $s$ "[All Fields] OR "crowned"[All Fields] OR "crowns"[MeSH Terms] OR "crowns"[All Fields] OR "crown"[All Fields]) AND (("stainless steel"[MeSH Terms] OR ("stainless"[All Fields] AND "steel"[All Fields]) OR "stainless steel"[All Fields]) AND ("crown $s$ "[All Fields] OR "crowned"[All Fields] OR "crowns"[MeSH Terms] OR "crowns"[All Fields] OR "crown"[All Fields])) AND (("primaries"[All Fields] OR "primary"[All Fields]) AND ("molar"[MeSH Terms] OR "molar"[All Fields] OR "molars"[All Fields] OR "molar s"[All Fields]))

\section{Study Selection}

Original studies reporting the clinical success of zirconia crowns and stainless steel crowns were included.

\section{Inclusion Criteria}

- Original studies of zirconia crowns comparing with stainless steel crowns.

- Studies conducted exclusively on primary molars.

- Follow up is more than one year.

- Studies published in English language.

\section{Exclusion Criteria}

- Animal studies.

- Case reports, case series.

- Studies in which sample size is less than 20.

- Studies conducted on permanent molars.

- Studies published in language other than English.

\section{Risk of Bias Assessment}

Two review authors independently graded all relevant articles in duplicate (Reviewer PS and SK). Risk of bias assessment was done using Cochrane risk of bias assessment tool. Articles were assessed on following domains: selection bias, reporting bias, attrition bias and others. Performance bias is not assessed as blinding of participants and personnel were not possible in the study. Reviewers assessed the domains in terms of 'low', 'unclear' or 'high'. The overall risk of bias was assessed considering the importance of different domains. Studies were classified as follows-'low risk of bias', 'unclear risk of bias' and 'high risk of bias'. Risk of bias assessment is summarized in Table 1.

\section{Statistical Analysis}

Meta-analysis was done using $\mathrm{R}$ programming. Packages used were Metafor, Meta, and qdap. Functions used were metaprop, forest and funnel. Random effects model were performed for zirconia and stainless steel groups as the data was found to be heterogeneous and were compared using subgroup analysis.

\section{Results}

The search yielded 4,609 potentially eligible articles which were evaluated independently by two reviewers (Reviewer PS and SK). First selection was done based on title and abstract, out of which 61 articles were considered for full-text evaluation. Selected articles were evaluated for further methodological appraisal based on inclusion and exclusion criteria. After final screening of the articles, seven randomized control trials were included in the review Table 2 .

Majority of the studies adopted split-mouth study design and the follow up period ranged from 12 months to 36 months. Sample size varied from 30 to 240 primary molars. Among the selected articles, only the study from Egypt, evaluated the radiographic success of the crowns. ${ }^{9}$ A Bulgarian study comparing the crown restoration was excluded from the review because of poor methodology and study design..$^{10}$ A study from Saudi Arabia was excluded as it assessed only the stainless steel crowns. ${ }^{11}$ Restorative success of the crowns and gingival health were the two outcomes measured in the review.

In a comparative study from Saudi Arabia, both zirconia and stainless steel crowns gave $100 \%$ success rates in terms of clinical 


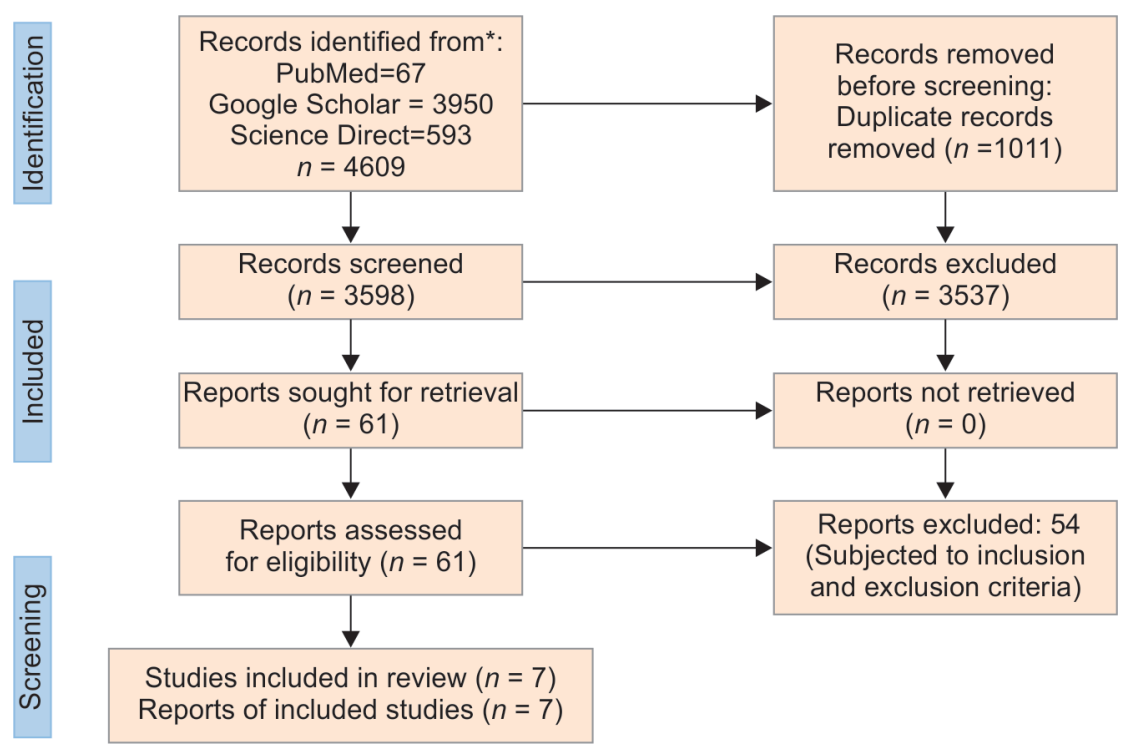

Fig. 1: Summary of article selection

Table 1: Risk of bias assessment

\begin{tabular}{|c|c|c|c|c|c|}
\hline \multicolumn{2}{|c|}{ Authors } & Random sequence generation & Allocation concealment & Incomplete outcome data & Operator bias \\
\hline \multicolumn{6}{|c|}{ Abdulhadi et al. $2017^{8}$} \\
\hline \multicolumn{6}{|c|}{ Abuelniel et al. $2018^{9}$} \\
\hline \multicolumn{6}{|c|}{ Donly et al. $2018^{12}$} \\
\hline \multicolumn{6}{|c|}{ Taran et al. $2018^{13}$} \\
\hline \multicolumn{6}{|c|}{ Mathew et al. $2020^{14}$} \\
\hline \multicolumn{6}{|c|}{ Donly et al. $2020^{15}$} \\
\hline \multicolumn{6}{|c|}{ Mathew et al. $2020^{16}$} \\
\hline Low risk & High risk & Unclear & & & \\
\hline No. & Authors name & Sample size & Follow-u & p period & \\
\hline 1. & Abdulhadi et al. $2017^{8}$ & 120 & 12 mont & $4-8 y$ & \\
\hline 2. & Abuelniel $2018^{9}$ & 240 & 12 mont & $4-6 y$ & \\
\hline 3. & Donly et al. $2018^{12}$ & 100 & 24 mont & $3-7 y$ & \\
\hline 4. & Taran et al. $2018^{13}$ & 30 & 12 mont & $6-9 y$ & \\
\hline 5. & Mathew et al. $2020^{14}$ & 60 & 36 mont & $6-8 y$ & \\
\hline 6. & Donly et al. $2020^{15}$ & 35 & 36 mont & $3-7 y$ & \\
\hline 7. & Mathew et al. $2020^{16}$ & 60 & 12 mont & $6-9 y$ & ars \\
\hline
\end{tabular}

success. None of the groups showed gingival bleeding when assessed during follow up. None of the zirconia group had plaque accumulation whereas $22 \%$ of stainless steel group showed the same. ${ }^{8}$

In an Egyptian study comparing the clinical and radiographic success of crowns in children aged 4-6 years over a period of 12 months, success rate was $75.8 \%$ for stainless steel crowns and $80.8 \%$ for zirconia crowns. Clinical success was evaluated based on length, position, polish and scratches. Radiographic success was assessed on smoothness and adaption of crown margins and the interproximal bone level. Gingival index and oral hygiene index were used to assess the gingival health following the placement of the crowns. Stainless steel crowns showed statistically significant higher gingival and oral hygiene index scores compared to zirconia crowns at the end of 12 months follow up period. They concluded that zirconia crowns showed favorable gingival health and acceptable clinical and radiographic success. ${ }^{9}$

An USA based 24 months randomized control study was conducted to evaluate the clinical success of primary zirconia molar crown with stainless steel crowns.100 primary molars with crowns were followed up for 6,12 and 24 months. Clinical success was assessed based on extent the crown was high in occlusion, staining on crown surface, wear of opposing arch tooth, surface roughness, color match, marginal integrity, anatomic form, marginal discoloration, proximal contact area, secondary caries at crown margin, and parent/guardian satisfaction with crown appearance. 
Gingival health was assessed using modified Loe and Sillness index. Crowns were categorized into clinically ideal, clinically acceptable and clinically unacceptable and none of the crowns were clinically unacceptable in the study. ${ }^{12}$

An in vivo study from Turkey compared the gingival health and clinical success of stainless steel crowns and zirconia crowns for a period of 12 months. Crown retention, gingival marginal extension, stain resistance, fracture were the clinical criteria. Gingival health of zirconia crowns was better in the study when compared to stainless steel crowns. The study showed stainless steel crowns was more retentive at the end of the follow up period. ${ }^{13}$ In a 36 month follow-up study from India, both stainless steel and zirconia crowns showed no plaque accumulation when evaluated at the end of follow up period. ${ }^{14}$

A split-mouth study in USA was conducted to evaluate the success of primary zirconia crowns and stainless steel crowns for 36 months. There were no failures in either of the groups and the results indicated zirconia crowns were as effective as stainless steel crowns, clinically. But zirconia crowns showed better gingival health and the results were statistically significant. ${ }^{15}$

A randomized control study in India was undertaken to evaluate Streptococcus mutans adhesion, plaque accumulation and gingival inflammation on zirconia and stainless steel crowns over a period of 12 months. Streptococcus mutans counts were higher in stainless steel group than zirconia group. The plaque accumulation and gingival inflammation was significantly more in stainless steel group when compared to the intervention group. ${ }^{16}$

Forest plot showed an overall proportion of 0.99 which was found to have no differences in the clinical success rates between zirconia and stainless steel among all the studies included. There was only one study from Egypt that showed a higher success rate for zirconia crowns (Fig. 2). A higher heterogeneity was found among the studies with a Chi-square value of $75 \%$.

\section{Discussion}

Stainless steel crown has better successful record of restoring the primary molars with added advantage of durability and pliability. However, the surface adjustment and manipulation of stainless steel crown cause defects and roughness on the surface accumulating more plaque. Zirconia crowns were introduced as an esthetic alternative to stainless steel crowns in pediatric dentistry. But, placing a crown in oral cavity will act as a niche for plaque adhesion and result in the development of secondary caries and affect the long term restorative success of crowns. ${ }^{16}$

\section{Gingival Health}

All the selected articles compared gingival health between stainless steel crowns and zirconia crowns. There is insufficient demographic data on which primary molars (first primary molars or second primary molars, maxillary primary molars or mandibular molars) the crowns were placed. However, the adoption of split-mouth study can reduce the bias. An Egyptian study and a study from Saudi Arabia had high risk of bias in the methodology as they lacked random allocation of the participants and moreover, oral hygiene instructions were not given and assessed gingival health as one of the primary outcomes.

Seven randomized control trials were included in the review with a follow-up from 12 months to 36 months. Gingival health of zirconia crowns was significantly better in case of inflammation and plaque accumulation when compared to stainless steel crowns. But, in a study from India there was no plaque accumulation on both zirconia and stainless steel crowns at the end of 36 months follow-up period. ${ }^{14}$ Six out of seven studies reported statistically significant difference in the gingival health scores between the groups. A study conducted in China revealed that, majority of stainless steel crowns had one or more defects in which crown crimping being the most common. But the plaque accumulation was less compared to control teeth in the study. ${ }^{17}$ A study from Saudi Arabia stated that oral hygiene level had a significant effect on the gingival index in patients with stainless steel crowns. ${ }^{11}$ The reason for less plaque accumulation in zirconia crowns may be attributed to glazed surfaces of crowns. ${ }^{18}$ The plaque accumulation was no longer present for stainless steel crowns as well as for zirconia crowns as the follow up period progressed. ${ }^{14}$ An Indian study evaluated Streptococcus colony counts on stainless steel crowns and zirconia crowns and found that the values was much lower on zirconia (3.3CFU) when compared to stainless steel crowns (45.16 CFU) and it was statistically significant. ${ }^{16}$ All the studies assessed the gingival health of the zirconia crowns over stainless steel crowns using different indices. Hence, quantitative assessment was not performed on the gingival health outcome of the crowns. Summary of evaluation of gingival health is given in Table 3.

\section{Restorative Success}

The operator had prepared the tooth for stainless steel crowns and zirconia crowns according to the manufacturer's instructions. In a systematic review, it was concluded that resin based cement were more appropriate for zirconia crowns cementation. ${ }^{19}$ Operators had used resin-based cements for luting the crowns. However, in an Indian study, they had cemented both the crown types using the type I glass ionomer cement. But, there was no clinical failure in terms of retention in both the groups after 36 months follow-up. ${ }^{14}$ Six articles were considered for meta-analysis and on the basis of risk of bias assessment, three articles were categorized as "low risk of bias" and three articles were categorized under "high risk of bias."

Studies evaluated success under the criteria of crown retention, stain resistance, occlusion, proximal contact, wear on opposing tooth, marginal integrity and discoloration and surface roughness. None of the studies showed statistically difference between the zirconia group and stainless steel group. An Egyptian study evaluated the radiographic success rate of zirconia and stainless steel groups. Stainless steel showed $9.2 \%$ of interproximal bone resorption whereas it was $7.5 \%$ for zirconia group and the findings were not statistically significant. ${ }^{9}$ Zirconia and stainless steel crowns were equally effective as a full crown restoration in the included studies. In fact, in one study from Turkey, the retention of stainless steel was better than zirconia crown. Minor staining and fracture of the surface of zirconia crowns were the main reason for failure in zirconia group. ${ }^{13}$ No secondary caries and wear of opposing teeth noted in both crown types. ${ }^{15}$ Results were summarized in Table 4.

Six studies were included in the meta-analysis and the funnel plot showed highly heterogeneous data among the studies (Fig. 2C). In the random effect model the proportion was 0.99 and showed no statistical significance (Fig. 2A). In the subgroup analysis, proportion of stainless steel crown was 1.00 under $95 \%$ confidence interval whereas it was 0.99 for zirconia crowns and were not statistically significant (Fig. 2B). A higher heterogeneity was found among the study which may be probably due to the difference in clinical parameters used to assess the restorative success of the crowns.

From the limited quality of evidence, it can be concluded that stainless steel crowns were equally effective as zirconia crowns when considering restorative success. 
Abdulhadi et al_2017_Saudi Arabia Abdulhadi et al 2017 Saudi Arabia Abuelniel and Ettawil_2018_Egypt Abuelniel and Ettawil_2018_Egypt

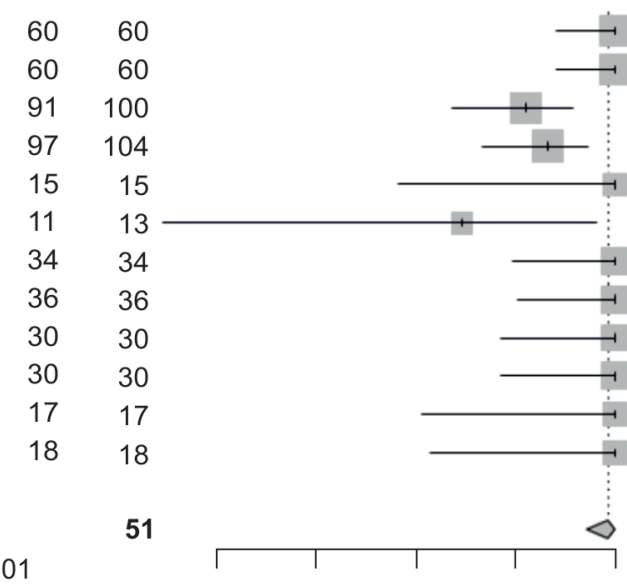

Heterogeneity: $\mid 2=75 \%, \square 2=0.0146, p<0.01$ A
Taran and Kaya_2018_Turkey

Donly et al_2018_USA

Donly et al_2018_USA

Mathew et al_2020 India

Mathew et al 2020 India

Donly et al_2020_USA

Donly et al_2020_USA
$1.00[0.94 ; 1.00]$

$1.00[0.94 ; 1.00]$

$0.91 \quad[0.84 ; 0.96]$

$0.93[0.87 ; 0.97]$

$1.00[0.78 ; 1.00]$

$0.85[0.55 ; 0.98]$

$1.00[0.90 ; 1.00]$

$1.00 \quad[0.90 ; 1.00]$

$1.00[0.88 ; 1.00]$

1.00 [0.88; 1.00$]$

$1.00[0.80 ; 1.00]$

$1.00[0.81 ; 1.00]$

$0.99[0.97 ; 1.00]$
$10.0 \%$

$10.0 \%$

$11.0 \%$

$11.0 \%$

$6.0 \%$

$5.5 \%$

$8.5 \%$

$8.7 \%$

$8.2 \%$

$8.2 \%$

$6.4 \%$

$6.6 \%$

$100.0 \%$
Study

CROWN TYPE = SS crowns

Abdulhadi et al_2017 Saudi Arabia Abuelniel and Ettawil_2018_Egypt

Taran and Kaya_2018_Turkey

Donly et al_2018_USA

Mathew et al_2020 India

Donly et al_2020_USA

Random effects model

Heterogeneity:

CROWN TYPE = Zirconia crowns Abdulhadi et al 2017 Saudi Arabia Abuelniel and Ettawil_2018_Egypt Taran and Kaya_2018_Turkey

Donly et al_2018_USA

Mathew et al_2020_India

Donly et al 2020_USA

Random effects model

Heterogeneity: $\mid 2=76 \%, \square 2=0.0180, p<0.0$

Random effects model

Heterogeneity: $\mathrm{I} 2=75 \%, 2=0.0146, p<0.01$
Events Total

$\begin{array}{lllll}0.6 & 0.7 & 0.8 & 0.9 & 1\end{array}$

F Proportion $\quad 95 \%-\mathrm{Cl}$ Weight

$1.00 \quad[0.94 ; 1.00] \quad 10.0 \%$

$0.91 \quad[0.84 ; 0.96] \quad 11.0 \%$

$1.00 \quad[0.78 ; 1.00] \quad 6.0 \%$

$1.00 \quad[0.90 ; 1.00] \quad 8.5 \%$

$1.00 \quad[0.88 ; 1.00] \quad 8.2 \%$

$1.00[0.80 ; 1.00] \quad 6.4 \%$

$1.00[0.96 ; 1.00] \quad 50.0 \%$

$1.00[0.94 ; 1.00] \quad 10.0 \%$

$0.93[0.87 ; 0.97] \quad 11.0 \%$

$0.85[0.55 ; 0.98] \quad 5.5 \%$

$1.00[0.90 ; 1.00] \quad 8.7 \%$

$1.00[0.88 ; 1.00] \quad 8.2 \%$

$1.00[0.81 ; 1.00] \quad 6.6 \%$

$0.99[0.95 ; 1.00] \quad 50.0 \%$

$0.99[0.97 ; 1.00] \quad 100.0 \%$

B

517

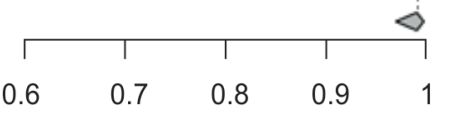

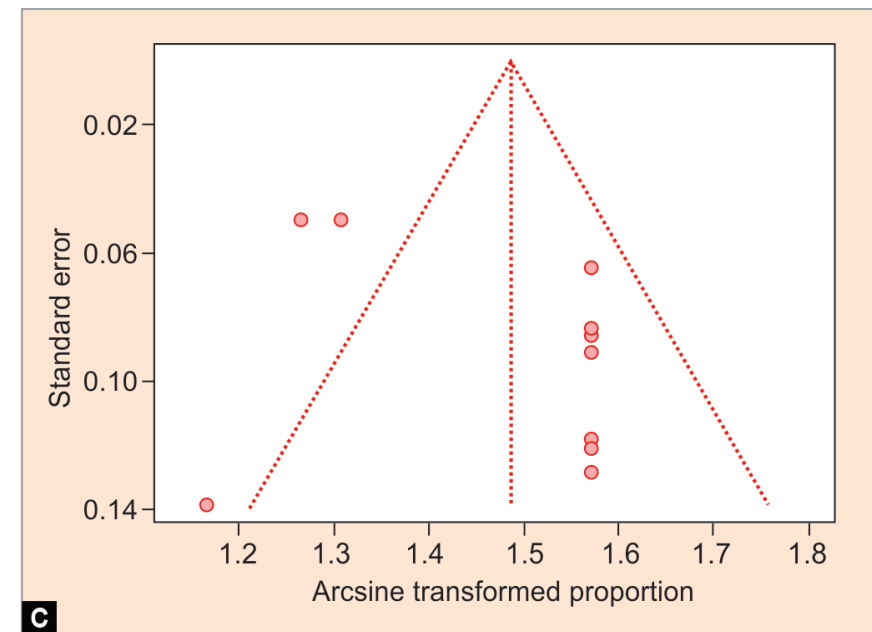

Figs 2A to C: (A) Statistical analysis of the group with random effects model, (B) Subgroup analysis between zirconia and stainless steel crowns, (C) Funnel plot showing heterogeneity 
Table 3: Summary of results of assessment of gingival health from the studies

\begin{tabular}{|c|c|c|}
\hline No. & Authors name & Gingival health \\
\hline 1. & $\begin{array}{l}\text { Abdulhadi et al. } \\
2017^{8}\end{array}$ & $\begin{array}{l}\text { No gingival bleeding } \\
\text { SS- } 100 \% \\
\text { Zirconia-100\% } \\
\text { No plaque accumulation- } \\
\text { SS-78\% } \\
\text { Zirconia-100\% }\end{array}$ \\
\hline 2. & $\begin{array}{l}\text { Abuelniel et al. } \\
2018^{9}\end{array}$ & $\begin{array}{l}\text { Gingival index } \\
\text { SS-0.62 } \\
\text { Zirconia-0.37 } \\
\text { OHI index } \\
\text { SS-0.63 } \\
\text { Zirconia-0.41 }\end{array}$ \\
\hline 3. & $\begin{array}{l}\text { Donly et al. } \\
2018^{12}\end{array}$ & $\begin{array}{l}\text { SS-76\% clinically ideal } \\
24 \% \text { clinically acceptable } \\
\text { Zirconia-78\% clinically ideal } \\
22 \% \text {-clinically acceptable }\end{array}$ \\
\hline 4. & $\begin{array}{l}\text { Taran et al. } \\
2018^{13}\end{array}$ & $\begin{array}{l}\text { Plaque index } \\
\text { SS-1 } \\
\text { Zirconia-0 } \\
\text { Gingival index } \\
\text { SS-0.25 } \\
\text { Zirconia-1 }\end{array}$ \\
\hline 5. & $\begin{array}{l}\text { Mathew et al. } \\
2020^{14}\end{array}$ & $\begin{array}{l}\text { SS-100 } \% \text { no plaque accumulation } \\
\text { zirconia- } 100 \% \text {, no plaque accumulation }\end{array}$ \\
\hline 6. & $\begin{array}{l}\text { Mathew et al. } \\
2020^{16}\end{array}$ & $\begin{array}{l}\text { Gingival index } \\
\text { SS-2.41 } \\
\text { Zirconia-1.01 } \\
\text { Plaque index } \\
\text { SS-2.11 } \\
\text { Zirconia-1.76 }\end{array}$ \\
\hline
\end{tabular}

(SS, stainless steel, $\mathrm{OHI}$, oral hygiene index)

Table 4: Summary of results of restorative success from the studies

\begin{tabular}{|c|c|c|c|}
\hline No. & Authors name & Clinical success & Radiographic success \\
\hline 1. & $\begin{array}{l}\text { Abdulhadi } \\
\text { et al. } 2017^{8}\end{array}$ & $\begin{array}{l}\text { SS crown-100\% } \\
\text { Zirconia crown-100\% }\end{array}$ & Not evaluated \\
\hline 2. & $\begin{array}{l}\text { Abuelniel et } \\
\text { al. } 2018^{9}\end{array}$ & $\begin{array}{l}\text { SS crown } \\
75.8 \% \\
\text { Zirconia crown } \\
80.8 \%\end{array}$ & $\begin{array}{l}\text { SS crown75.8\% } \\
\text { Zirconia crown } \\
80.8 \%\end{array}$ \\
\hline 3. & $\begin{array}{l}\text { Donly et al. } \\
2018^{12}\end{array}$ & $\begin{array}{l}\text { SS crown-100\% } \\
\text { Zirconia crown-100\% } \\
\text { (none of them were } \\
\text { clinically unacceptable) }\end{array}$ & Not evaluated \\
\hline 4. & $\begin{array}{l}\text { Taran et al. } \\
2018^{13}\end{array}$ & $\begin{array}{l}\text { SS crown- } 100 \% \\
\text { Zirconia crown-84.6\% }\end{array}$ & Not evaluated \\
\hline 5. & $\begin{array}{l}\text { Mathew et al. } \\
2020^{14}\end{array}$ & $\begin{array}{l}\text { SS crown-100\% } \\
\text { Zirconia crown-100\% }\end{array}$ & Not evaluated \\
\hline 6. & $\begin{array}{l}\text { Donly et al. } \\
2020^{15}\end{array}$ & $\begin{array}{l}\text { SS crown-100\% } \\
\text { Zirconia crown-100\% }\end{array}$ & Not evaluated \\
\hline 7. & $\begin{array}{l}\text { Mathew et al. } \\
2020^{16}\end{array}$ & Not evaluated & Not evaluated \\
\hline
\end{tabular}

\section{Limitations}

Authors have used various indices to evaluate the gingival health, hence the comparison of zirconia and stainless steel couldn't be done quantitatively. Secondly, due to lack of insufficient data on radiographic outcome of these crowns, it was not assessed in the current review.

\section{Conclusion}

In this review we reached the following conclusion:

- Gingival health was better for zirconia crowns when compared to stainless steel crowns. However, more qualitative and quantitative assessment is recommended.

- From the limited quality of evidence, stainless steel crowns were equally effective as zirconia crowns.

- More studies are warranted with longer follow-ups and radiographic comparison between stainless steel crowns and zirconia crowns.

\section{References}

1. Sheller B, Williams BJ, Lombardi SM. Diagnosis and treatment of dental caries-related emergencies in a children's hospital. Pediatr Dent 1997;19 (8):470-475. PMID: 9442540.

2. McDonald RE, Avery DR, Dean JA. Restorative dentistry. In: McDonald RE, editor. Dentistry for the Child and Adolescent. $9^{\text {th }}$ ed. Missouri: Mosby Inc.; 2011. pp. 333-339.

3. Randall RC. Preformed metal crowns for primary and permanent molar teeth: review of the literature. Pediatr Dent 2002; 24:489-500. PMID: 12412964.

4. Tinanoff N, Douglass JM. Clinical decision-making for caries management in primary teeth J Dent Educ 2001;65(10): 1133-1142. DOI: $10.1002 / j .0022-0337.2001 .65 .10 . t b 03462 . x$

5. Piconic Maccaur G. Zirconia as ceramic biomaterial. Biomaterials 1999; 20(1): 1e25. DOI: 10.1016/s0142-9612(98)00010-6

6. Abd El-Ghany OS, Sherief AH. Zirconia based ceramics, some clinical and biological aspects. Future Dent J 2016; 2(2):55-64. DOI: org/10.1016/j.fdj.2016.10.002

7. Planells Del Pozo P, Fuks AB. Zirconia crowns-an esthetic and resistant restorative alternative for ECC affected primary teeth. J Clin Pediatr Dent 2014; 38(3):193-195. DOI: 10.17796/jcpd.38.3.0255q84jt2851311

8. Abdulhadi BS, Abdullah MM, Alaki SM, et al. Clinical evaluation between zirconia crowns and stainless steel crowns in primary molars teeth. J Pediatr Dent 2017; 5(1):21. DOI: 10.4103/jpd.jpd_21_17

9. Abuelniel G, Eltawil S. Clinical and radiographic evaluation of stainless steel versus zirconia crowns on primary molars: randomized controlled trial. Egypt Dent J 2018; 64(2):977-989. DOI: 10.21608/ edj.2018.76940

10. Dimitrova D, Andreeva R, Dimova-Gabrovska M. Study of the gingival health in children with crown restorations. Scr Sci Med Dent 2018; 4(1):28-31.

11. Sharaf AA, Farsi NM. A clinical and radiographic evaluation of stainless steel crowns for primary molars. J Dent 2004; 32(1):27-33. DOI: 10.1016/s0300-5712(03)00136-2

12. Donly KJ, Sasa I, Contreras $\mathrm{Cl}$, et al. Prospective randomized clinical trial of primary molar crowns: 24-month results. Pediatr Dent 2018; 40(4):253-258. PMID: 30345963.

13. Taran PK, Kaya MS. A comparison of periodontal health in primary molars restored with prefabricated stainless steel and zirconia crowns. Pediatr Dent 2018; 40(5):334-339. PMID: 30355428.

14. Mathew MG, Roopa KB, Soni AJ, et al. Evaluation of clinical success, parental and child satisfaction of stainless steel crowns and zirconia crowns in primary molars. J Fam Med Prim Care 2020; 9(3):1418. DOI: 10.4103/jfmpc.jfmpc_1006_19

15. Donly KJ, Méndez MJC, Contreras $\mathrm{Cl}$, et al. Prospective randomized clinical trial of primary molar crowns: 36 -month results. Am J Dent 2020; 33(3):165-168. PMID: 32470244.

16. Mathew MG, Samuel SR, Soni AJ, et al. Evaluation of adhesion of Streptococcus mutans plaque accumulation on zirconia and stainless steel crowns, and surrounding gingival inflammation in primary molars: randomized controlled trial. Clin Oral Investig 2020; 18: 1-6. DOI: $10.1007 / \mathrm{s} 00784-020-03204-9$ 
17. Chao DD, Tsai TP, Chen TC. Clinical evaluation of gingival tissue restored with stainless steel crown. Changgeng Yi Xue Za Zhi 1992; 15(4):198. PMID: 1295654.

18. Daou EE. The zirconia ceramic: strengths and weaknesses. Open Dent J 2014; 8:33. DOI: 10.2174/1874210601408010033
19. Gargari M, Gloria F, Napoli E, et al. Zirconia: cementation of prosthetic restorations. Literature review. Oral Implantol 2010; 3(4):25. PMID: 23285393. 